\title{
From nuclei to atoms and molecules: the chemical history of the early Universe
}

\author{
dedicated to Pr. Dennis William Sciama \\ Denis Puy ${ }^{\dagger, \ddagger}$, Monique Signore ${ }^{\star}$ \\ ${ }^{\dagger}$ Institute of Theoretical Physik, University of Zurich (Switzerland) \\ $\ddagger$ Paul Scherrer Institut, Villigen (Switzerland) \\ ${ }^{\star}$ Laboratoire de Radioastronomie, Observatoire de Paris (France)
}

\begin{abstract}
The dark age of the Universe is generally pointed out as the period between the recombination epoch $(z \sim 1000)$ and the horizon of current observations $(z \sim$ $5-6)$. The arrow of time in the cosmic history describes the progression from simplicity to complexity, because the present Universe is clumpy and complicated unlike the homogeneous early Universe. Thus it is crucial to know the nature of the constituents, in order to understand the conditions of the formation of the first bound objects.

In this paper we analyse the chemical history of this dark age through the creation of the primordial nuclei to the formation of the first atoms and molecules. Then we will describe the consequences of the molecular formation on the birth of the protoobjects. In this context we will mention the important works of Dennis W. Sciama who influenced a large number of theorists -cosmologists and astronomers- on this new field of research dedicated to primordial molecules.
\end{abstract}

Key words: Early Universe

PACS: 01.30

\section{Introduction}

At early times the Universe was filled up with an extremely dense and hot gas. Various important physical processes occurred as a consequence of the expansion. For example, Universe cooled below the binding energies of light elements leading to the formation of the nuclei which recombined with the

1 E-mail: puy@physik.unizh.ch, monique.signore@obspm.fr 
electrons in order to form the first atoms. After recombination, although the density decrease acts against molecular formation, it turns out that the temperature is small enough for this formation occurs.

The existence of a significant abundance of molecules can be crucial on the dynamical evolution of collapsing objects. Because the cloud temperature increases with contraction, a cooling mechanism can be important for the structure formation, by lowering pressure opposing gravity, i.e. allowing continued collapse of Jeans unstable protoclouds. This is particularly true for the first generation of objects.

Interactions between primordial molecules and the cosmic microwave background radiation (CMBR) could be important if the molecular abundances are sufficient (Dubrovich 1977, Melchiorri \& Melchiorri 1994, Signore et al. 1997). In particular, a resonant molecular scattering between CMBR photons and molecules could, on one hand, smear out primary CMBR anisotropies and, on the other hand, produce secondary anisotropies.

In this paper we will describe the chemical history of the Universe from the formation of the first atoms until the formation of the first objects. Thus in Sect. 2 we will recall the nucleosynthesis then the important influence of the neutrinos physics in Sect. 3. The period of recombination will be described in Sect. 4 which will lead to the description of the chemical chemistry or formation of primordial molecules in Sect.5. We will discuss in Sect.6 the influence of the molecules on the formation of the first objects, and on the CMBR in Sect.7. The possible reionization of the Universe could be some importance consequences on the chemical history of the Universe, in Sect.8 we will describe it, and we will give some conclusions and prospects in Sect.9.

\section{Primordial nucleosynthesis}

The Standard Big Bang Nucleosynthesis model (SBBN) has been analysed first by Peebles (1966), Wagoner, Fowler \& Hoyle (1967), Wagoner (1969, 1973). Nevertheless the Chicago Group gave strong impulse to this field, see Yang et al. (1979, 1984), Copi et al (1995), Schramm \& Turner (1998), Schramm (1998) and Olive et al. (1981, 2000), and the Oxford Pole led by Sarkar (1996, 1999). Here we only summarize the main features of the SBBN model that we have already presented elsewhere (Signore \& Puy 1999).

The standard model of the very early Universe is quite simple and determined by the three important milestones of the early Universe: the expansion governed by General Relativity, the particle interactions governed by Standard Model and particle distribution governed by statistical physics. The SBBN depends on just one parameter which is the baryon-to-photon ratio $\eta$ or the dimensionless quantity of baryon density $\Omega_{b}$.

In the framework of the SBBN model at times much less than one second 
after the big bang, the Universe was a hot $\left(T>>10^{10} \mathrm{~K}\right)$ rapidly expanding plasma, where most of its energy is on the form of photons at high temperature and relativistic particles. The weak processes such as:

$$
n+\nu \longleftrightarrow p+e^{-}, n+e^{+} \longleftrightarrow p+\nu, \text { and } n \longleftrightarrow p+e^{-}+\bar{\nu}
$$

maintained the ratio of neutrons to protons at its thermal value, i.e. $n / p \sim 1$ (where $n$ define the neutrons, $p$ the protons, $e^{-}$the electrons, $e^{+}$the positrons and $\nu$ the neutrinos).

At about one second, the temperature of the universe is around $10^{10} \mathrm{~K}$, the above weak processes became ineffective. The $n / p$ ratio froze out at about $1 / 6$, leading to the collisions between neutrons and protons which form deuterium:

$$
n+p \longrightarrow D+\gamma
$$

The collisions with deuterium led to the formation of the helium 3 and tritium nuclei:

$$
n+D \longrightarrow{ }^{3} H, \quad p+D \longrightarrow{ }^{3} \mathrm{He},
$$

and finally to ${ }^{4} \mathrm{He}$ via the reactions:

$$
n+{ }^{3} \mathrm{He} \longrightarrow{ }^{4} \mathrm{He}, \quad p+{ }^{3} \mathrm{H} \longrightarrow{ }^{4} \mathrm{He}, \mathrm{D}+\mathrm{D} \longrightarrow{ }^{4} \mathrm{He} .
$$

At about 100 sec. the nucleosynthesis epoch was over, thus most neutrons were in ${ }^{4} \mathrm{He}$ nuclei, and most protons remained free while smaller amounts of $\mathrm{D},{ }^{3} \mathrm{He}$ and ${ }^{7} \mathrm{Li}$ were synthetized. The low densities, the Coulomb barriers and stability gaps at masses 5 and 8 worked against the formation of heavier elements.

The chemical composition of the Universe remains unchanged until the formation of the first stars. The yields of primordial nucleosynthesis are shown as a function of the baryon density in Fig.1 (from Burles et al. 1999).

It is a considerable challenge to measure the actual primordial abundances because of uncertainties in measuring present day abundances and because of uncertainties in modeling the nuclear evolution since the big bang. The measured abundances of helium 4, deuterium, lithium 7 and helium 3 (Burles et al. 1999) are:

$$
\begin{aligned}
Y_{p} & =0.244 \pm 0.002 \\
(D / H)_{p} & =(3.4 \pm 0.3) \times 10^{-5} \\
\left({ }^{7} \mathrm{Li} / H\right)_{p} & =(1.7 \pm 0.15) \times 10^{-10} \\
\left({ }^{3} \mathrm{He} / \mathrm{H}\right)_{p} & =(0.3 \pm 1) \times 10^{-5}
\end{aligned}
$$

Fig.1 summarizes the measured abundances with their uncertainties. Since 1980 and until recently, cosmologists introduced a concordance interval for the baryon density where the predicted and measured abundances for all the 
four light elements are consistent; see for instance Copi et al. (1995) who derived

$$
0.007<\Omega_{b} h^{2}<0.024
$$

A change occured in 1998-1999 with the accurate determination of the primordial deuterium abundance which led to the most accurate determination of the baryon density: Fig.1 shows the concordance intervals for each element and the baryon density predicted by the deuterium measurement (vertical band). From the latest determined primordial $D$ abundance (Tytler et al. 2000):

$$
(D / H)_{p}=(3.3 \pm 0.5) \times 10^{-5} \quad(95 \% \text { c.l. }) .
$$

Burles et al. (2000) give the following precise determination of the baryon density:

$$
\Omega_{b} h^{2}=0.0189 \pm 0.0019 \quad(95 \% \text { c.l. }) \text {. }
$$

Until this year, the agreement between the SBBN predictions and observed abundances makes the SBBN a cornerstone of the Big Bang cosmology But the recent cosmic microwave background measurements of BOOMERANG and MAXIMA (de Bernardis et al. 2000, Jaffe et al. 2000) favor the following value:

$$
\Omega_{b} h^{2}=0.030 \pm 0.032_{-0.008}^{+0.009} \quad(95 \% \text { c.l. })
$$

which, a priori, cannot be accomodated within the SBBN model (Burles et al. 2000). Future satellite experiments (MAP \& PLANCK surveyor) will give us a better cosmic microwave background determination of $\Omega_{b} h^{2}$.

Meanwhile, the SBBN model seems to be in trouble... and cosmologists search for non-standard cosmology or non standard big bang nucleosynthesis.

\section{$3 \quad$ Neutrinos and nucleosynthesis}

In cosmology, neutrinos have a strong impact on big bang nucleosynthesis and therefore important consequences on the chemistry of the universe. Recently an extensive litterature discussed the possible generation of sterile neutrinos in the early universe as a result of their oscillations with active neutrinos.

Dennis W. Sciama was not only concerned with active neutrinos and decaying neutrinos since a long time, but also with sterile neutrinos. In an European conference (Sciama 1999), he gave a review and pointed out the importance of this field ${ }^{2}$ and described the more recent developments on the sterile neutrinos and its relevance for both SBBN and the CMBR.

Thus, we briefly recall how SBBN can constrain the properties of active neutrinos and in particular how helium abundance can be used to count neutrinos.

2 he argued: students of the CMBR have now to learn something new, thanks to the famous announcement by the SuperKamiokande team as Neutrino 98, confirming the existence of an atmospheric neutrino anomaly... (see Sciama 99) 


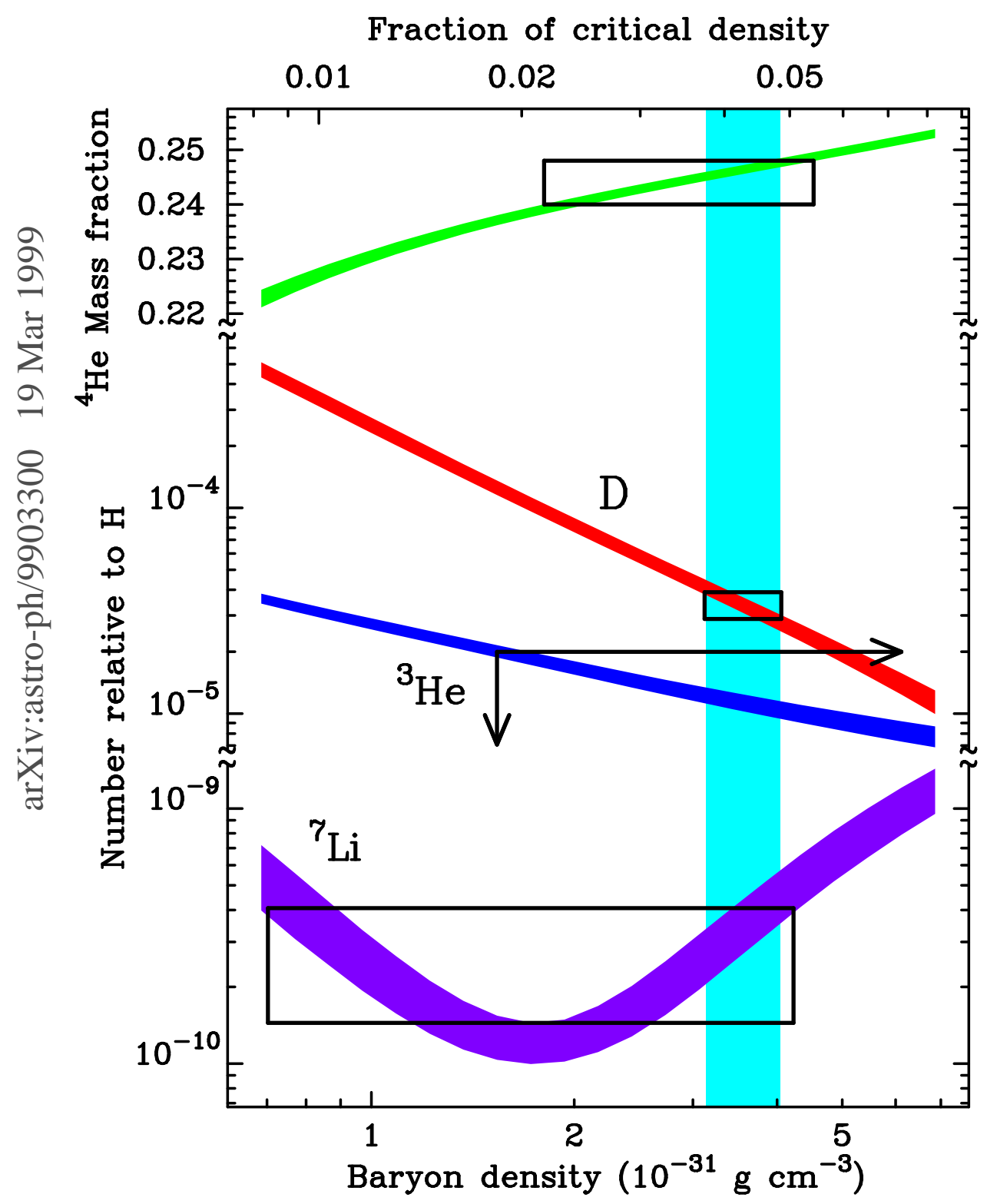

Fig. 1. : Relative abundances, by number: A/H, except for ${ }^{4} \mathrm{He}$ in mass fraction: $Y_{p}$. Concordance intervals for each element ( $2 \sigma$ uncertainty) and baryon density predicted (vertical line) by $D$ measurements are also shown (from Burles et al. 1999, astro-ph/9903300).

\subsection{Limits to the number of neutrinos}

In the standard model, neutrinos possess only weak interactions, and are coupled to the intermediate bosons $Z^{o}$. Measurements of the bosonic decay width allow to conclude that the total number of different neutrino species is

$$
N_{\nu}=3.07 \pm 0.12
$$


while the combined fit to LEP data gives the more accurate results:

$$
N_{\nu}=2.994 \pm 0.01
$$

In the framework of the SBBN model the precise epoch of nucleosynthesis was controlled by the number of particle degrees of freedom: more neutrino species imply a higher energy density and a faster expansion, i.e. more neutrons and more ${ }^{4} \mathrm{He}$ production. Therefore the ${ }^{4} \mathrm{He}$ abundance can be used to constrain the number of neutrinos species. For instance, Steigman et al. (1977) gave the SBBN limit of 7 neutrino species while the laboratory limit was about 5000 ! At this time, the SBBN limit was a very good constraint.

The helium curve in Fig.1 was computed assuming $N_{\nu}=3$. Burles et al. (2000) get the upper limit $N \nu<3.2$ while Lisi et al. (1999) give $N_{\nu}<4$ depending on what observational constraints one uses.

More generally, the limits on $N_{\nu}$ can be translated into limits on other types of particles (numbers or masses) that would affect the expansion rate just before the nucleosynthesis.

\subsection{Sterile neutrinos}

Sterile neutrinos are neutrinos which lack the standard electroweak interactions possessed by the active neutrinos: $\nu_{e}, \nu_{\mu}$ and $\nu_{\tau}$. In particular, they do not contribute to the decay width of $Z^{o}$; they would gravitate; therefore, if they existed in the early universe, their gravitational effects would influence the rate of expansion and therefore also the SBBN.

Different studies have been recently done and showed the role played by neutrino oscillations in SBBN; in particular, there are some controversies about the possible generation of lepton asymmetry by oscillations between active and sterile neutrinos and its consequences on the observed baryon asymmetry of the universe, on primordial abundances (especially ${ }^{4} \mathrm{He}$ and also $\mathrm{D}$ ), on the CMBR (Hannestad \& Raffelt 1999) and on the decaying neutrinos (see later in section 8). Look, for instance: Bell et al. (1998), Sciama (1998, 1999), Mohapatra \& Sciama (1998), Dolgov (2000) and references therein.

\subsection{BOOMERANG, MAXIMA and the number of neutrinos}

As already said in Sect.2, the pre-BOOMERANG and MAXIMA consensus for the central value for $\Omega_{b} h^{2}$ deduced from SBBN is

$$
\Omega_{b} h^{2}=0.0189
$$


while the central value implied by BOOMERANG and MAXIMA measurements (Jaffe et al. 2000) is:

$$
\Omega_{b} h^{2}=0.03 .
$$

The difference between the SBBN and the cosmic microwave background central values for $\Omega_{b} h^{2}$ has already triggered discussions, explanations in a lot of papers. Among them: Hu et al. (2000), Tegmark \& Zaldarriaga (2000), White et al. (2000), Kinney et al. (2000), Kurki-Suonio \& Sihvola (2000), KurkiSuonio (2000), Hannestad et al. (2000) and Melchiorri \& Griffiths (2000).

Let us only mention that an alternative solution can be lepton asymmetry; for instance, Esposito et al. (2000) find the best fit to the BOOMERANG and MAXIMA data with $N_{\nu}=9$ or suggest the best compromise $N_{\nu}=13$ for cosmic microwave background data and light elements abundances; Lesgourgues \& Peloso (2000) find a reasonable fit with $\Omega_{b} h^{2}=0.028$ and an effective number of neutrinos $N_{\nu}=6$. Active-sterile neutrino oscillations can also explain the current observations of cosmic microwave background and light elements abundances (di Bari \& Foot 2000). BOOMERANG and MAXIMA data and the number of neutrinos are a very active field of study.

\section{Recombination epoch}

In the hot Big Bang picture, after the nucleosynthesis period the natural question concerns the possibility to form atoms by recombination of primordial nucleus with free electrons, and the second question concerns the degree to which the recombination is inhibited by the presence of recombination radiation.

The reason for which these questions are central were mentionned by Peebles (1968) and Peebles \& Dicke (1968) based, at this epoch, on the primevalfireball picture of Gamow (1948) for the evolution of the Universe.

The cosmological recombination process was not instantaneous because the electrons, captured into different atomic energy levels, could not cascade instantaneously down to the ground state. Atoms reached the ground state either through the cosmological redshifting of the Lyman $\alpha$ line photons or by the $2 s-1 s$ two photons process. Nevertheless the Universe expanded and cooled faster than recombination could completed, and small fraction of free electrons and protons remained.

\subsection{Recombination of Hydrogen}

The principles of calculations of the primordial recombination have been mentionned initially by Shklovskii (1967), Novikov \& Zel'dovich (1967) and Takeda \& Sato (1968). Peebles (1968) was the first to present a theory in which the 
very complicated recombination process is reduced to simpler terms ${ }^{3}$. The methodology of the calculations consist to take into account a three level atom with a ground state, first excited state and continuum, represented by a recombination coefficient. A single ordinary differential equation is derived to describe the ionisation fraction (see Peebles 1968). The assumptions are:

- Hydrogen excited states are in equilibrium with the radiation

- Stimulated de-excitation is negligible

- Collisional processes are negligible

The approximations are based on the rate of transfer of energy per unit volume between radiation and free electrons developped by Kompaneets (1957) then Weymann $(1965,1966)$ and coupled with the expansion of the Universe.

Peebles (1968) computed the rate of recombination of the plasma from the rate coefficient for recombination to excited states of hydrogen tabulated by Boardman (1964), and showed that the residual ionization of the hydrogen (or fractional ionization $x_{e}$ ) is below $5.3 \times 10^{-5}$ for a flat cosmological model at redshift below 1500. Jones \& Wyse (1985) developped two important analytic approximations: one, valid at the redshift $z>1500$ is a modification of the standard Saha equation, and the other, valid for $800<z<1500$. They found that the fractional ionization is roughly $6.75 \times 10^{-4}$ at the redshift $z=555$ much higher than the Peebles values. More recently Seager, Sasselov \& Scott $(1999,2000)$ have presented a refined treatment of the recombination through a complete code which performs appproximate calculations history ${ }^{4}$.

\subsection{Recombination of Helium}

We have seen that ${ }^{4} \mathrm{He}$ is the second most abundant element in the Universe after hydrogen. Zel'dovich, Kurt \& Sunyaev (1968) and Matsuda, Sato \& Takeda (1969) were the first to point out that the cosmological recombination of helium differs from that of hydrogen. The conditions for the helium recombination are such that in both cases it occurs in accordance with the Saha equation (Bernshtein \& Dubrovich 1977, Dubrovich \& Stolyarov 1997). Recombination history of early Universe consist of three stages but helium recombination occurs in two steps:

- at $z \sim 6000$ (or when the temperature became less than $16000 \mathrm{~K}$ ) the

3 by means of a number of approximations valid in the conditions of interest, so that the physical situation can be described by a few variables, and the recombination equation can be integrated by a few variables, and the recombination equation can be integrated by hand, without having to abandon any of the essential elements of the problem as written by Peebles himself in 1968.

4 see http://www.astro.ubc.ca/people/scott/recfast.html. 
first electron recombines and singly ionized $\mathrm{HeII}$ forms from doubly ionized HeIII.

- Then at $z \sim 2700$ (or temperature close to $7300 \mathrm{~K}$ ), HeII recombines into a neutral state.

- from $z \sim 1500$ recombination of hydrogen took place.

More recently Seager, Sasselov \& Scott (2000) have developped an improved recombination calculation of $H, H e$ (with $H e I I$ and $H e I I I)$ that involves a lineby-line treatment of each atomic level. They found that $\mathrm{HeI}$ recombination is much slower than previously thought, and it is delayed until just before $H$ recombines.

\subsection{Recombination of Lithium}

The ionization potential $I_{L i}=5.392 \mathrm{eV}$ of $L i$ I is shorter than that of hydrogen $\left(I_{H}=13.6 \mathrm{eV}\right)$. Dalgarno \& Lepp (1987) suggested the existence of $\mathrm{Li}^{+}$ions after the period of the hydrogen recombination, and showed that the radiative recombination is again effective at the redshift $z \sim 450$. This last point is particularly important for the lithium chemistry as we will see.

\section{$5 \quad$ Primordial chemistry}

From the pioneer works of Zwicky (1959) on the molecular hydrogen content of the Universe, presented at the San Francisco meeting of the Astronomical Society of the Pacific in June 1959, the idea of molecular formation at the immediate post-recombination took form.

The studies on the primordial chemistry (or post-recombination chemistry) have been the source of a tremendous increase of the litterature. The first complete description of the hydrogen chemistry was developped by different japanese groups: Hirasawa, Aizu \& Taketani (1969), Takeda, Sato \& Matsuda (1969) and Matsuda, Sato \& Takeda (1969) in the context of pre-galactic gas clouds or formation of galaxies. The importance of the deuterium chemistry through the formation of the $H D$ molecule was suggested by Lepp \& Shull (1983) as lithium chemistry through the formation of the molecule $\mathrm{LiH}$, completed by the works of Dalgarno \& Lepp (1987) on the helium chemistry and the lithium chemistry. Latter a complete comprehensive chemistry of the lithium, at the post-recombination epoch, was presented by Stancil, Lepp \& Shull (1996).

The first chemical network including the primordial molecules (such as $H_{2}$, $H D$ and $L i H$ ) and ions was carried out by Lepp \& Shull (1984), Latter \& Black (1991), Puy et al. (1993) and more recently by Galli \& Palla (1998), 
and Stancil, Lepp \& Dalgarno (1998). The development of the primordial chemistry studies owe to Dalgarno an important contribution concerning the calculations of the reaction rates (see Lepp \& Shull 1983, Dalgarno \& Lepp 1987, Puy et al. 1993 and Galli \& Palla 1998 and references therein).

\subsection{Hydrogen chemistry}

After the important works of the recombination period, it was plausible to imagine that molecular hydrogen plays a role in the early evolution of large clouds. Nevertheless $\mathrm{H}_{2}$ was not considered as a component of the pre-galactic medium.

In a review article dedicated to the hydrogen molecules in astronomy Field, Somerville \& Dressler (1966), showed that the times scales for appreciable amounts to form in three-body reactions or in radiative association were always greater than the Hubble age. In the cosmological context there are no grains which can catalyze the reaction of formation, thus any $\mathrm{H}_{2}$ formed in the uniform background is dissociated by the radiation, until the density is too low to produce it. Saslaw ${ }^{5} \&$ Zipoy (1969) then Shchekinov \& Entél (1983) showed the importance of the charge transfer reactions:

$$
H_{2}^{+}+H \rightarrow H_{2}+H^{+}
$$

initiated by the radiation association

$$
H+H^{+} \rightarrow H_{2}^{+}+h \nu
$$

They pointed out that $H_{2}^{+}$ion is converted to $H_{2}$ as soon as it is formed and the $H_{2}^{+}$concentration never becomes large.

Peebles \& Dicke (1968), in a scenario concerning the origin of the globular star clusters, showed that these clusters may have originated as gravitationnally bound gas clouds before the galaxies formed, and suggested that some molecular hydrogen can form, mainly by way of negative hydrogen by the reactions (see also Shchekinov \& Entél 1983)

$$
H+e^{-} \rightarrow H^{-}+h \nu
$$

followed by the reaction

$$
H^{-}+H \rightarrow H_{2}+e^{-}
$$

5 In 1967, William C. Saslaw was a Ph.D. student of Dennis W. Sciama at the Department of Applied Mathematics and Theoretical Physics of the University of Cambridge (see the family tree in Ellis et al. 1993) 
with the reaction rates calculated by Mc Dowell (1961) from remarks on the quantum theory of the negative hydrogen ion emphasized by Chandrasekhar (1944), in which the electrons act only as catalysts. Thus two possible ways of formation were pointed out.

Takeda, Sato \& Matsuda (1969) were the firsts to study the evolution of molecular hydrogen abundance in the cosmological medium (i.e. post-recombination Universe) in contrast with the works by Saslaw \& Zipoy (1967) and Peebles and Dicke (1968) in which they calculated the products of $H_{2}$ in dense clouds. They considered the two ways of $H_{2}$ formation and electron and proton as a kind of catalyzer.

The photodetachment of $\mathrm{H}^{-}$and the photodissociation of $H_{2}^{+}$by the background radiation field restrict the abundance of molecular hydrogen formed at early stages, although the photodestruction of molecular hydrogen is negligible. The destruction is due to collisional dissociation (Lepp \& Shull 1983).

\subsection{Deuterium chemistry}

Deuterium chemistry in a early Universe could play an important role in the sense that it could give some explanations on the controversy observations of fractional abundance in high redshift Ly $\alpha$ (Songaila et al. 1994, Burles \& Tytler 1996). Although similar processes contribute to the formation of $H D$ :

$$
\begin{aligned}
& D^{+}+H \rightarrow H D^{+}+h \nu \\
& H D^{+}+H \rightarrow H^{+}+H_{2},
\end{aligned}
$$

its formation also proceeds through:

$$
\begin{gathered}
H^{+}+D \rightarrow D^{+}+H \\
D^{+}+H_{2} \rightarrow H^{+}+H D .
\end{gathered}
$$

Thus the formation of $H D$ is carried out in two steps. Nevertheless when the abundance of $\mathrm{H}_{2}$ is sufficient, the second way of formation is dominant (Eq. 8). Stancil, Lepp \& Dalgarno (1998) presented a complete review of the deuterium chemistry of the early Universe.

\subsection{Helium chemistry}

The presence of helium in the early Universe gives rise to a rich assembly of molecular processes as pointed out by Hirasawa (1969). The main molecular 
species containing helium is $\mathrm{HeH}^{+}$. Processes that lead to the formation and destruction of the molecular ion $\mathrm{HeH}^{+}$in astrophysical environments have been the subject of a number of investigations. Dabrowski \& Herzberg (1978) were the one of the firsts to introduce the possibility that this molecular ion exists in astrophysical plasmas; it is from these initial works that Roberge \& Dalgarno (1982) presented the formation and destruction mechanisms. More recently Zygelman, Stancil \& Dalgarno (1998) calculated the enhancement of the rate coefficient for the radiative association to form $\mathrm{HeH}^{+}$:

$$
\mathrm{He}+\mathrm{H}^{+} \rightarrow \mathrm{HeH}^{+}+\nu
$$

The $\mathrm{H}_{2}^{+}$ions and $\mathrm{H}_{2}$ produce $\mathrm{HeH}^{+}$by the fast reactions

$$
\begin{aligned}
& \mathrm{H}_{2}^{+}+\mathrm{He} \rightarrow \mathrm{HeH}^{+}+\mathrm{H}, \\
& \mathrm{H}_{2}+\mathrm{He}^{+} \rightarrow \mathrm{HeH}^{+}+\mathrm{H} .
\end{aligned}
$$

Nevertheless for all of these processes, once photodissociation ceases to be rapid, the $\mathrm{H}_{2}^{+}$ions react preferentially with neutral atomic hydrogen to form molecular hydrogen as we have seen.

\subsection{Lithium chemistry}

The lithium chemistry is initiated by the recombination of lithium, which occured near the redshift $z \sim 450$. The formation of the molecular ion $\mathrm{LiH}^{+}$ formed by radiative association processes:

$$
\begin{gathered}
L i^{+}+H \rightarrow L i H^{+}+h \nu \\
H^{+}+L i \rightarrow L i H^{+}+h \nu
\end{gathered}
$$

opens the way of the formation of the $\mathrm{LiH}$ molecules through the exchange reactions

$$
\mathrm{LiH}^{+}+\mathrm{H} \rightarrow \mathrm{LiH}+\mathrm{H}^{+}
$$

which are more rapid than the formation by radiative association of $H$ and $L i$ atoms.

The complete description of the lithium chemistry was emphasized by Stancil, Lepp \& Dalgarno (1996) where new quantal rate coefficients were included. 


\subsection{Molecular abundances}

In order to estimate the molecular abundances which depend on the reaction rates ${ }^{6}$, it is necessary to know the thermal and dynamical evolution of the medium. In the Einstein-de Sitter Universe the evolution of matter temperature $T_{m}$ is described by the equation:

$$
\frac{d T_{m}}{d t}=\alpha x_{e} T_{r}\left(T_{r}-T_{m}\right)-\frac{2 T_{m}}{a} \frac{d a}{d t}
$$

where $\alpha$ is a constant, $x_{e}$ the fractional ionization of the hydrogen, $T_{r}$ the temperature of the cosmological radiation temperature and $a(t)$ the expansion parameter. The first term describes the heat transfer from radiation to the electrons when the second term characterizes the cooling due to the expansion of the Universe. The result of the integration of this important equation led to the idea that there is thermal decoupling between matter and radiation. Nevertheless the formation of primordial molecules such as $\mathrm{H}_{2}, \mathrm{HD}$, and $\mathrm{LiH}$ can involve a thermal response due to the excitation of the rotational levels of these molecules. The population of the rotational levels is mainly due to collisional excitation and de-excitation with $\mathrm{H}, \mathrm{H}_{2}$ and $\mathrm{He}$ on one hand and to radiative processes on the other hand: absorption from the Cosmic Microwave Background Radiation (CMBR) and spontaneous or induced emission.

The importance of radiative cooling rate by molecular hydrogen were pointed out by Takayanagi \& Nishimura (1960), which developped theoretical calculations of the cooling process due to the rotational excitation of $\mathrm{H}_{2}$ molecule. They considered collisions with hydrogen atom in the context of interstellar clouds. Saslaw \& Zipoy (1967) used the radiative function of Takayanagi \& Nishimura (1960) in the context of pre-galactic gas clouds. The molecules $H D$ and $\mathrm{LiH}$ play an important thermal role, since their allowed dipole rotational transitions, see Lepp \& Shull (1984). Puy et al. (1993) calculated precisely the population of the levels and provide a molecular thermal function $\Psi_{\text {mol }}$ due to $\mathrm{H}_{2}, \mathrm{HD}$ and $\mathrm{LiH}$. A complete description of the cooling of astrophysical media is found for $\mathrm{H}_{2}$ in Le Bourlot et al. (1999), and for $H D$ in Flower et al. (2000). Flower (2000) presented recently a study on the role of $H D$ in the thermal balance of the primordial gas and conclude that $H D$ is about as important as $\mathrm{H}_{2}$ in the thermal balance of the primordial gas. Notice that the estimation of the ortho-para $\mathrm{H}_{2}$ in the primordial gives a better estimation of the $H_{2}$ cooling as Flower \& Pineau des Forêts (2000) emphasized very recently. Furthermore some chemical reactions produce a chemical thermal function $\Theta_{\text {chem }}$ through the enthalpy of reaction.

6 the complete chemical network is described in Puy et al. (1993), Galli \& Palla (1998) and Stancil et al. (1998). 
In the cosmological context the thermal evolution equation becomes

$$
\frac{d T_{m}}{d t}=\alpha x_{e} T_{r}\left(T_{r}-T_{m}\right)-\frac{2 T_{m}}{a} \frac{d a}{d t}+\frac{2 \Psi_{m o l}}{3 n k}+\frac{2 \Theta_{c h e m}}{3 n k}
$$

Puy et al. (1993) took into account the transfer process between radiation and matter via the Thomson scattering, coupled with the molecular source term and the enthalpy of the reactions in order to calculate the abundances of the species $^{7}$.

After a transient growth all abundances of the molecules become almost constant. The final abundances of $H_{2}, H D$ and $L i H$ freeze out due to the expansion. Moreover the final values of molecular abundances depend on the choice of the cosmological parameters. Galli \& Palla (1998) summarized and discussed the chemistry of early Universe for different cosmological models. In the standard model defined by the following cosmological parameters:

$H_{o}=67$ for the Hubble constant and $\eta_{10}=4.5$ for the baryon-to-photon ratio in an Einstein-de Sitter Universe. In this context we have at the end of the recombination, the following initial abundance:

$D / H \sim 4.3 \times 10^{-5}$, and $L i / H \sim 2.2 \times 10^{-10}$.

In this context we obtained the following final abundance at $z=5$ :

$H_{2} / H \sim 1.1 \times 10^{-6}, H D / H \sim 1.2 \times 10^{-9}$ and $L i H / H \sim 7.2 \times 10^{-20}$

and for the main molecular ions with $x_{e} \sim 3 \times 10^{-4}$ :

$H_{2}^{+} / H \sim 1.3 \times 10^{-12}, H D^{+} / H \sim 2.1 \times 10^{-18}, H_{2} D^{+} / H \sim 5.1 \times 10^{-14}$, $\mathrm{HeH}^{+} / \mathrm{H} \sim 6.2 \times 10^{-13}$ and $\mathrm{LiH}^{+} / \mathrm{H} \sim 9.4 \times 10^{-18}$.

\section{Primordial objects}

The developement of structure in the universe was well advanced at high redshifts. For example, quasars have been detected nearly to $z \sim 5$, and the most distant galaxies to even greater distances. Still unknown, however, is the epoch during which the first generation of objects was formed. Hoyle (1953) were one of the firsts to suggest that the importance of the fragmentation process of extragalactic hydrogen cloud gas clouds, with particular reference to the formation of galaxies out of hot hydrogen clouds. From these pioneer

7 The numerical integration of the coupled chemical equation is an initial value problem for stiff differential equations (see Puy et al. 1993). 
works Hunter (1962) developped an instability theory of a collapsing gas cloud. Gravitational instability and thermal instability were supposed to be the main processes to form condensations in a dilute gas. In the framework of the gravitational instability theory, each structure started as a tiny local overdensity; nevertheless very little is known about the protoclouds. Fluctuations that survive decoupling are subject to gravitational instabilities if they are on sufficiently large scale. The general treatment of the gravitational instability in an expanding Universe, developped by Lifshitz (1946) and Lifshitz \& Khalatnikov (1963), showed that a condensation due to gravitational instability cannot grow so fast during the time scale of the Universe. Parker (1953) suggested that the problem of condensation can be understood as a consequence of instability on the thermal equilibrium of a diffuse medium.

Since the works of Jeans (1902) the importance of thermal instability as another possible mechanism for galaxy formation were made by Hoyle (1958) and above all Field (1965). Kato, Nariai \& Tomita (1967) examined the thermal instability of a dilute gas in expanding Universe. Sofue (1969) found that formation of galaxies could be initiated by thermal instability due to radiative cooling, and developped by Saito (1969) in the context of a uniformly rotating homogeneous medium. The formalism were definitively established by Kondo (1970) in an expanding medium.

Doroshkevish, Zel'dovich \& Novikov (1967) showed that if the cloud contracts adiabatically, hydrogen atoms in it are soon collisionally ionized again and the cloud is finally dispersed by radiation pressure. Therefore, some kind of cryogen is necessary to form a bound system as mentionned Takeda, Sato \& Matsuda (1968). The problem was to find a good cryogen and so primordial molecules offered an interesting solution. Matsuda, Sato \& Takeda (1969) realized that hydrogen molecule could be a possible cooling of pre-galactic gas clouds from the works on the $\mathrm{H}_{2}$ cooling in the interstellar clouds of Takayanagi \& Nishimura (1960). Yoneyama (1972) pointed out that after hydrogen molecules are formed, the cooling of the gas by these molecules plays an important role on the further condensation and fragmentation of the cloud. Since these first works, the hypothesis that galaxies formed from density perturbations which collapse within the expanding background of a Friedmann universe appears worthy of continued investigation. Nevertheless all of these first models considered the collapse of non-rotating spherical clouds. Thus Hutchins (1976) studied the thermal effects of $H_{2}$ molecules in rotating and collapsing spheroidal gas clouds, and suggested an early period of formation of objects in the mass range of ordinary stars; which led to the model of Silk (1977) concerning the fragmentation of cosmic gas clouds consisting of gas of a predominantly primordial composition. The contraction of a such cloud is initially adiabatic.

Early studies focused on the chemical evolution and cooling of primordial clouds by solving a chemical reaction network within highly idealized collapse models (Palla et al. 1983, Mac Low \& Shull 1986, Puy \& Signore 1996, Tegmark et al. 1997). Some hydrodynamic aspects were studied in spherical 
symmetry and multidimensional studies by Anninos \& Norman (1996) and more recently by Abel et al. (2000).

A small fractional abundance of $H_{2}$ molecules is formed via the $H^{-}$way. The small residual ionization remaining after decoupling is sufficient to produce enough $\mathrm{H}_{2}$ to radiate away the compressional energy of collapse. Further collapse proceeds almost isothermally, and considerable fragmentation occurs. This process is terminated only when at sufficiently high densities the $\mathrm{H}_{2}$ molecules are destroyed by the reaction:

$$
H_{2}+H+H \rightarrow 3 H
$$

Palla, Salpeter \& Stahler (1983) examined precisely the three body reactions

$$
\begin{gathered}
H+H+H \rightarrow H_{2}+H \\
H+H+H_{2} \rightarrow H_{2}+H_{2},
\end{gathered}
$$

and investigated the thermal and chemical evolution of a collapsing spherical cloud composed of pure hydrogen gas, and various regimes of oscillation in the collapse can occurs as Lahav (1986) showed.

Lepp \& Shull (1984) emphasized that the dipole rotational transitions of $H D$ and $L i H$ are particularly important at high density and low temperature. In this context Puy \& Signore (1997) examined the evolution of primordial molecules in a context of gravitational collapse and showed how the abundances can be modified. The importance of $H D$ molecules was pointed out by Puy \& Signore (1998b) where they analysed the ratio between the molecular cooling due to $H D$ and that due to $H_{2}$, and found that the main cooling agent around $200 \mathrm{~K}$ is $H D$. This results was confirmed by Okumurai (2000), Uehara \& Inutsuka (2000) and Flower et al. (2000).

The cooling of LiH is less important. Lepp \& Shull (1984) and Puy \& Signore (1996) considered the $\mathrm{LiH}$ molecules formed through only the radiative association. Stancil, Lepp \& Dalgarno (1996), Dalgarno, Kirby \& Stancil (1996) and Gianturco \& Gori-Giorgi (1996) proposed a new lithium chemistry with new ways of $\mathrm{LiH}$ formation. In this context Bougleux \& Galli (1997) then Puy \& Signore (1998a) developped a complete lithium chemistry in a collapsing cloud. Nevertheless at the temperature higher than $200 \mathrm{~K}, \mathrm{H}_{2}$ and $H D$ cooling are thermically dominant as Abel et al. (1997) pointed out in threedimensional numerical simulations, which lead to a scenario of the formation of first stars in the Universe due to the fragmentation of primordial gas (see also Bromm, Coppi \& Larson 1999).

Thus primordial molecules play an important role in the pregalatic gas particularly on the thermochemical stability as mentionned by Rozenzweig et al. (1994) and on the problem of the minimum mass which virialized in order to form the first cosmological objects (Tegmark et al. 1997). 


\section{Anisotropies of CMBR and primordial molecules}

The study of the CMB anisotropies (CMBA) -primary and secondary onesare important tools to study the origin and the evolution of perturbations. Different physical processes are responsible for the production of the CMBA: each one associated with a typical angular scale.

Among the different kinds of secondary CMBA, the Rees-Sciama effect (Rees \& Sciama 1968) can produce a detectable signal only if very large masses are involved $\left(M>10^{16} \mathrm{M} \odot\right)$ and can give significant contributions only at large angular scales (degree scale) and in $\Omega \neq 1$ scenarii. The Sunyaev-Zel'dovich effect (Sunyaev \& Zel'dovich 1972) is important at cluster scales (subarcminute scale). At very small scales (subarcminute scale) two kinds of secondary CMBA can be sizeable: thermal emission by dust in galaxies and primordial molecular lines produced by resonant elastic scattering. Here, we only consider the primordial molecular lines.

\subsection{Interaction between the $C M B$ and primordial molecules}

From an initial idea of Zel'dovich, Dubrovich (1977) showed that resonant elastic scattering must be considered as the most efficient process in coupling matter and radiation at high redshift. They noted that the cross section for resonant scattering between $\mathrm{CMB}$ and molecules is several orders of magnitude larger than that between $\mathrm{CMB}$ and electrons: even a modest abundance of primordial molecules would produce significant Thomson scattering. At this point, every velocity field (due to molecular motion or to cloud infall) would leave its imprint on CMB via Doppler shift. This technique for exploring the early universe has been revised by the group of Melchiorri (De Bernardis et al. 1993, Melchiorri \& Melchiorri 1994, Maoli et al. 1994, Signore et al. 1994). A careful analysis of various primordial molecules has led to the identification of $\mathrm{LiH}$ and $\mathrm{LiH}^{+}$as the best candidates (Dubrovich 1977, de Bernardis 1993). Note only that this conclusion was relevant because all these authors assumed a value for the $\mathrm{LiH}$ abundance (i.e. $\mathrm{LiH} / \mathrm{H} \sim 3 \times 10^{-8}-3 \times 10^{-9}$ ) which was adopted -at that time- by all the experts in primordial chemistry.

An attempt to search for $\mathrm{LiH}$ has even been carried out with the IRAM $30 \mathrm{~m}$ telescope (de Bernardis et al. 1993). Because the resonant scattering is an elastic process, it can result in a primary CMBA attenuation and in a secondary CMBA production, exactly as in the case of an early reionisation of the uni-

verse. Note that this effect is strongly frequency-dependent while the effect of Thomson scattering in a ionized medium is not frequency dependent. Finally, one can show that the blurring of primary CMBA by resonant scattering lines of $\mathrm{LiH}$ can occur if $\mathrm{LiH} / \mathrm{H}>3 \times 10^{-8}$ for $\nu<60 \mathrm{GHz}$ and for intermediate angular scales (see Maoli et al. 1994). 


\subsection{Possible molecular signals?}

Just as primary CMBA could be erased, secondary CMBA could be expected by molecular resonant scattering. In the framework of a Cold Dark Matter (CDM) scenario -and also in a more general case- Maoli et al. (1996) have calculated the intensity the line width of the expected signal during the three stages of the evolution of a very simple protocloud model: linear phase, turnaround epoch and non-linear collapse. In particular, they have shown that for standard observational conditions $\left(10^{-5}<\Delta \nu / \nu<10^{-4} ; \Delta I / I_{C B R}>10^{-4}\right.$ the beginning of the non-linear collapse phase of a protocloud, just after its turnaround epoch, is the best one for a detection of the first two LiH rotational lines which are observable in the frequency range $30<\nu<250 \mathrm{GHz}$ with an angular scale range $7 "<\theta<20$ ". They concluded that the IRAM 30-m telescope with its 10" of angular resolution and its frequency channels, is well suited for this kind of observations, see also Signore et al. (1997).

\subsection{Discussion}

The effects of $\mathrm{LiH}$ molecules on the CMBA -erasing of primary CMBA, creation of secondary CMBA- strongly depend on the final $\mathrm{LiH}$ abundance which is a function of the lithium produced by primordial nucleosynthesis and of the efficiency of its conversion to $\mathrm{LiH}$. With the quantal rate coefficient for the radiative association of lithium and hydrogen given by Stancil et al. (1996) (see Sect.6) the fractional abundance of $\mathrm{LiH}$ is much smaller than that of the above CMBA studies and therefore leads to no erasing of the primary CMBA and no production of secondary CMBA. But let us only emphasize that, again at present times, the primordial lithium abundance and the percentage of lithium converted to $\mathrm{LiH}$ are both quite uncertain !

\section{Reionization}

In the 1960's years there were several ways which led to the idea that the medium at high redshift could be ionized: The radiation from a very distant object could be scattered by free electrons (Field 1954, Gunn \& Peterson 1965); radio waves of low frequency from extragalactic sources would be absorbed (Field 1954, Sciama 1964a, Ericson \& Cronyn 1965), and the radiation from radio sources could suffer dispersion (Haddock and Sciama 1965). Sciama (1964a, 1964b) and thereafter Rees \& Sciama (1966) were one of the first to put forward that the intergalactic hydrogen could be partially ionized at large scale. 
The absence of a Gunn-Peterson (Gunn \& Peterson 1965) effect in the spectra of high redshift quasars implies that the intergalactic medium is highly ionized at low redshifts (i.e. $z \sim 5$ ). These observations suggested that the intergalactic medium was reionized in the redshift interval $(5<z<300)^{8}$. Thus at epochs corresponding to $z \sim 1000$ the intergalactic medium is expected to recombine and remain neutral until sources of radiation and heat develop that are able of reionizing it.

A number of suggestions have been made for possible alternative sources of intergalactic ionizing photons. Sciama (1993a) gave an exhaustive description of the scenario of these alternatives sources.

The scenario through decay of dark matter particles has been proposed by different authors, the first was pointed by Cowsik (1977) and extented by de Rujula \& Glashow (1980) from massive neutrinos decayed into lighter neutrinos and UV photons. The hypothesis of the photoionization of the intergalatic medium by radiatively decaying neutrinos or photinos has been previously formulated by Sciama (1982a, 1982b, 1982c), which led also to a scenario describing the ionization of $H I$ regions in the galaxy (Sciama 1990a) or the ionization of Hydrogen throughout the Universe (Sciama 1990b, 1993b, 1994, Sethi 1997). In this context Salati \& Wallet (1984) reinvestigated the recombination of neutral hydrogen taking into account light neutral fermions, stable or radiatively unstable. They found a limit on the fractional ionization:

$4 \times 10^{-4}<x_{e}<2 \times 10^{-3}$

instead of the previous baryon-dominated universe result:

$3 \times 10^{-5}<x_{e}<3 \times 10^{-4}$.

Sciama (1988) from the works of Cabibbo, Farrar, Maiani (1981), Sciama \& Melott (1982) and Sciama (1982c, 1984) proposed a scenario of photino decay in order to explain the observation of the ionization of Lyman- $\alpha$ clouds at large redshifts.

Bowyer et al. (1999) observed the spectrum of the night sky with an extremeultraviolet spectrometer covering the bandpass from 350 to $1100 \AA$ on board the spanish satellite MINISAT-1 launched in 1997 April 21. The observed is far less intense than that expected in the Sciama model of radiative decay of massive neutrinos, but they cannot exclude the earlier model of Melott (1984), based also on the theory of Sciama with a decay energy which is somewhat greater than $13 \mathrm{eV}$, and different than the $13.6 \mathrm{KeV}$ of the Sciama model.

\section{Conclusions}

First, let us recall that the latest $\mathrm{CMB}$ results require a higher baryon density than allowed by SBBN theory and observations and therefore new physics -for

8 The detection of CMBR anisotropies rules out a fully ionized intergalactic medium beyond $z \sim 300$, see Scott, Silk \& White (1995) 
example a strong lepton asymmetry-

Then, we have seen that the chemistry could have consequences on the formation of the proto-objects. Nevertheless many important questions still await answers. In primordial chemistry a lot of reaction rates are estimated only and particularly those involved in the formation of $\mathrm{LiH}$ molecules. Recently Dickinson \& Gadea (2000) developped a fully quantal description of the radiative association in $\mathrm{Li}^{+}+H$ collisions, and showed that the rate coefficient is at least five orders of magnitude larger than that for the classical radiative association $\mathrm{Li}+\mathrm{H}$. Thus the radiative cooling due to $\mathrm{LiH}$ could be changed ( $\mathrm{LiH}$ has a strong dipole moment).

Calculation of the rate coefficients, for rotational transitions induced in collisions between the primordial molecules and atoms, is crucial. For example Roueff \& Zeippen (1999) presented calculations of rotational excitation of $H D$ molecules by $H e$ atoms, and provide a new estimation of molecular cooling and heating. In this context Le Bourlot et al. (1999) and Flower et al. (2000) showed the importance to use the recent quantum mechanical calculations of cross-sections for rotational transitions, in order to estimate respectively the $\mathrm{H}_{2}$ cooling and $H D$ cooling. Thus recently the rate coefficients are accessed in the software library of Collaborative Computational Project ${ }^{9}$, the UMIST data-base ${ }^{10}$ which provide a large data of reaction rates and collisional coefficients. Primordial chemistry is still in a nascent stage: the development of computing technics and specifical software for the quantum mechanics will open large possibilities.

Primordial molecules could play a role at the turn-around period, i.e. where the expansion of the density perturbation is maximum and begins to collapse, and a modification of the temperature at this turn-around point can occur (Puy 2000b, Puy \& Signore 2001). Moreover the primordial molecules play a crucial role on the evolution of the first objects, through the process of fragmentation. The first objects could be massive stars. Thus, as soon as stellar processes occur, proto-objects can lead through SN explosions to the formation of other molecules species, such as $C O, C I$ or $H C N$. They in turn are important sources of contamination of the medium, and thus can offer different ways of early pre-biotic molecular formation. Chakrabarti \& Chakrabarti (2000) showed that a significant amount of adenine $\left(H_{5} C_{5} N_{5}\right)$, a DNA base, may be produced during molecular cloud collapse through the chain reaction of $H C N$ addition. The existence of primordial carbon or nitrogen could also produce primordial bio-molecules or pre-biotic molecules just after the cosmological recombination period (see Puy 2000a).

The researches and the perpectives on primordial molecules are very large. The ESA cornerstone mission as Far InfraRed and Submillimetre Telescope (FIRST), or the Atacama Large Millimetre Array project (or ALMA), with a collecting area of up $10000 \mathrm{~m}^{2}$, will offer interesting perspective of detection.

$\overline{9}$ http://ccp7.dur/ac.uk/

${ }^{10}$ http://saturn/ma.umist.ac.uk:8000/ 


\section{Acknowledgements}

One of the authors (D.P.) was EEC Marie Curie Post-Doc Fellowship (19941996) at the International School of Advanced Studies (SISSA-Trieste) on the supervision of Dennis W. Sciama. Dennis W. Sciama gave complete freedom to choose the topics, and always followed this field with constant support and encouragement. The multiple discussions with Dennis W. Sciama showed that a great physicist have not only a formidable knowledge of the physics, but also a real sense of the human respect.

This works have been supported by the Dr Tomalla Foundation and by the Swiss National Science Foundation.

He was a man, take him for all in all. I shall not look upon this like again...

(Hamlet, William Shakespeare)

\section{References}

Abel T., Anninos P., Zhang Y., Normal M. 1997 New Ast. 3, 181

Abel T., Bryan G., Norman M. 2000 ApJ 540, 39

Aninos P., Norman M. 1996 ApJ 460, 556

Bell N. et al. 1998 Phys. Rev. D58, 5010

Bernshtein I., Bernshtein D., Dubrovich V. 1977 Astron. Zh. 54, 727

Boardman W. 1964 ApJ Suppl. 9, 185

Bougleux E., Galli D. 1997 MNRAS 288, 638

Bowyer S, Korpela E., Edelstein J. et al. 1999 ApJ 526, 10

Bromm V., Coppi P., Larson R. 1999 ApJ 527, L5

Burles S., Tytler D. 1996 ApJ 114, 1330

Burles S., Nollett K., Turner M. 1999, astro-ph/9903300

Burles S., Nollett K.,Turner M. 2000, astro-ph/0008495

Cabibbo N., Farrar G., Maiani L. 1981 Phys. Lett. B 105, 155

Chakrabarti S., Chakrabarti S.K. 2000 A\&A 354, L6

Chandrasekhar S. 1944 ApJ 100, 176

Copi C., Schramm D., Turner M. 1995 Science 267, 192

Cowsik R. 1977 Phys. Rev. Lett. 39, 784

Dabrowski I., Herzberg G. 1978 Ann. NY Acad. Sci. 38, 14

Dalgarno A., Lepp S. 1987 in Astrochemistry, ed. Vardya and Tarafdar, DordrechtReidel Eds

Dalgarno A., Kirby K., Stancil P. 1996 ApJ 458, 397

De Bernardis P. et al. 1993 A\&A 271, 683

De Bernardis P. et al. 2000 Nature 404, 955 
De Rujula A., Glashow S. 1980 Phys. Rev. Lett. 45, 942

Di Bari P., Foot R. 2000 astro-ph/0008258

Dickinson A., Gadea F. 2000 MNRAS 318, 1227

Dolgov A. 2000 hep-ph/0004032

Doroshkevich A., Zel'dovich Ya., Novikov I. 1967 Soviet Aj. 11, 233

Dubrovich V. 1977 Sov. Astr. Lett. 3, 128

Dubrovich V., Stolyarov V. 1997 Astron. Lett. 23, 565

Ellis G., Lanza A., Miller J., in The renaissance of General Relativity and Cosmology, Cambridge Univ. Press 1993

Ericson W., Cronyn W. 1965 ApJ 142, 1156

Esposito S. et al. 2000 astro-ph/0007419

Field G. 1959 ApJ 129, 536

Field G. 1965 ApJ 142, 531

Field G., Somerville W., Dressler K. 1966 Ann. Rev. Astron. Ap. 4, 207

Flower D., Le Bourlot J., Pineau des Forêts G., Roueff E. 2000 MNRAS 314, 753

Flower D. 2000 MNRAS 318, 875

Flower D., Pineau des Forêts G. 2000 MNRAS 3116, 901

Galli D., Palla F. 1998 A\&A 335, 403

Gamow G. 1948 Phys. Rev. 74, 505

Gianturco F., Gori-Giorgi P. 1996 Phys. Rev. A 54, 1

Gunn J., Peterson B. 1965 ApJ 142, 1633

Haddock F., Sciama D.W. 1965 Phys. Rev. Lett. 14, 1007

Hannestad S., Raffelt G. 1999 Phys. Rev. D59, 3001

Hannestad S., Hansen S., Villante F. 2000 astro-ph/0012009

Hirasawa T., Aizu K., Taketani M. 1969 Prog. Theor. Phys. 41, 835

Hirasawa T. 1969 Prog. Theor. Phys. 42, 523

Hoyle F. 1953 ApJ 118, 513

Hoyle F. 1958 proceedings of the 11th Solvay congress

$\mathrm{Hu}$ W. et al. 2000 astro-ph/0006436

Hunter C. 1962 ApJ 136, 594

Hutchins J. 1976 ApJ 205, 103

Jaffe A. et al. 2000 astro-ph/0007333

Jeans J. 1902 Phil. Trans. Roy. Soc. 199A, 49

Jones B., Wyse R. 1985 A\&A 149, 144

Kato S., Nariai H., Tomita K. 1967 Pub. Astron. Soc. Japan 19, 130

Kinney W., Melchiorri A., Riotto A. 2000 astro-ph/0007375

Kompaneets A. 1957, Soviet Phys. JETP 4, 730

Kondo M. 1970 Pub. Astr. Soc. Japan 22, 23

Kurki-Suonio H. 2000 astro-ph/0002071

Kurki-Suonio H., Sihvola E. 2000 astro-ph/0011544

Lahav O. 1986 MNRAS 220, 259

Latter W., Black J. 1991 ApJ 372, 161

Le Bourlot J., Pineau des Forets G., Flower D. 1999 MNRAS 305, 892

Lepp S., Shull M. 1983 ApJ 270, 578

Lepp S., Shull M. 1984 ApJ 280, 465 
Lesgourgues J., Peloso M. 2000 astro-ph/0004412

Lifshitz E. 1946 J. Phys. USSR 10, 116

Lifshitz E., Khalatnikov I. 1963 Advances in Phys. 12, 185

Lisi E., Sarkar S., Villante F. 1999 Phys. Rev. D59, 3520

Mac Dowell M. 1961, Observatory 81, 240

Mac Low M., Shull M. 1986 ApJ 302, 585

Maoli R. et al. 1994 ApJ 425, 372

Maoli R. et al. 1996 ApJ 457, 1

Matsuda T., Sato H., Takeda H. 1969 Prog. Theor. Theor. 41, 219

Melchiorri B., Melchiorri F. 1994 Riv. Nuovo Cim. 17, 1

Melchiorri A., Griffiths L. 2000 astro-ph/0011147

Melott 1984 Soviet Astron. 28, 478.

Mohapatra R., Sciama D.W. 1998 astro-ph/9811446

Novikov I., Zel'dovich Ya. 1967 Ann. Rev. Astr. Ap. 5, 627

Olive K. et al. 1981 ApJ 246, 557

Olive K. et al. 2000 Phys. Rep. 333, 389

Okumurai K. 2000 ApJ 534, 809

Palla F., Salpeter E., Stahler S. 1983 ApJ 271, 632

Parker E. 1953 ApJ 117, 431

Peebles P.J.E. 1966 Phys. Rev. Lett. 16, 410

Peebles P.J.E., Dicke R. 1968 ApJ 154, 891

Puy D. et al. 1993 A\&A 267, 337

Puy D., Signore M. 1996 A\&A 305, 371

Puy D., Signore M. 1997 New Ast. 2, 299

Puy D., Signore M. 1998a New Ast. 3, 27

Puy D., Signore M. 1998b New Ast. 3, 247

Puy D. 2000a A\&A in preparation, see astro-ph/0011496

Puy D. 2000b, in Proceedings $6^{\text {th }}$ Trieste Conference, astro-ph/0011435

Puy D., Signore M. 2001, in Primordial cosmochemistry, Nova Eds

Rees M., Sciama D.W. 1966 ApJ 145, 6

Rees M., Sciama D.W. 1968 Nature 517, 611

Roberge W., Dalgarno A. 1982 ApJ 255, 489

Rosenzweig P., Parravano A., Ibanez M., Izotov Y. 1994 ApJ 432, 485

Roueff E., Zeippen C. 1999 A\&A 343, 1005

Saito M. 1969 Pub. Astr. Soc. Japan 21, 230

Salati P., Wallet J.C. 1984 Phys. Lett. B 144, 61

Sarkar S. 1996, Rep Prog. Phys. 59, 1493

Sarkar S. 1999, astro-ph 9903183

Saslaw W., Zipoy D. 1967 Nature 216, 967

Schneider D., Schmidt M., Gunn J. 1991 ApJ 102, 837

Schramm D. 1998 Proc. Nat. Acad. Sci USA 95, 42

Schramm D., Turner M. 1998 Rev. Mod. Phys. 70, 303

Sciama D.W. 1964a Quart. Journ. R. Astr. Soc. 5, 196

Sciama D.W. 1964b Nature 201, 767

Sciama D.W. 1982a MNRAS 198, 1 
Sciama D.W. 1982b MNRAS 200, 13

Sciama D.W. 1982c Phys. Lett. B 114, 19

Sciama D.W. 1984 in Big Bang: G. Lemaitre in a modern world, ed Berger A., Dordrecht-Reidel Eds

Sciama D.W. 1988 MNRAS 230, 13p

Sciama D.W. 1990a ApJ 364, 549

Sciama D.W. 1990b Comments Astrophys. 15, 71

Sciama D.W. 1993a in Modern cosmology and the dark matter problem, Cambridge University Press

Sciama D.W. 1993b ApJ 409, L25

Sciama D.W. 1994 Phil. Trans. R. Soc. Lond. A 346, 137

Sciama D.W. 1998 astro-ph/9811172

Sciama D.W. 1999 in: $3 K$ Cosmology, Eds Maiani et al., AIP Conf Proc. 476, 74

Sciama D.W., Rees M. 1966 Nature 212, 1001

Sciama D.W., Melott 1982 MNRAS Phys. Rev. D 25, 2214

Scott D., Silk J., White S. 1995 Science 268, 829

Seager S., Sasselov D., Scott D. 1999 ApJ 523, L1

Seager S., Sasselov D., Scott D. 2000 ApJ 128, 407

Sethi S. 1997 ApJ 474, 13

Shchekinov Y., Entél M. 1983 SvA 27, 622

Shklovskii I 1967, in Proceedings $4^{\text {th }}$ Texas Conf. on Relativistic Ap. 5, 627

Signore M. et al. 1994 ApJ supp 92, 535

Signore M. et al. 1997 Astr. Lett. Com. 35, 349

Signore M., Puy D. 1999 New Ast. Rev. 43, 18

Silk J. 1977 ApJ 211, 638

Sofue Y. 1969 Pub. Astr. Soc. Japan 21, 211

Songaila A., Cowie L., Hogan C., Rugers M. 1994 Nature 368, 599

Stancil P., Lepp S., Dalgarno A. 1996 ApJ 472, 102

Stancil P., Lepp S., Dalgarno A. 1998 ApJ 509, 1

Steigman G. et al. 1977 Phys. Lett. B66, 202

Sunyaev R., Zel'dovich Y. 1972 Comments Astroph. Space Phys. 4, 173

Takayanagi K., Nishimura S. 1960 Pub. Astr. Soc. Jap. 12, 77

Takeda H, Sato H. 1968 in Proceedings of symposium on cosmology held at Kyoto February 1968 (in japanese), p 135

Takeda H., Sato H., Matsuda T. 1969 Prog. Theor. Theor. Phys. 41840

Tegmark M., Silk J., Rees M., Blanchard A., Abel T., Palla F. 1997 ApJ 474, 1

Tegmark M., Zaldarriaga M. 2000 astro-ph/0004393

Tytler D. et al. 2000 astro-ph/0001318

Uehara H., Inutsuka S. 2000 ApJ 531, L91

Wagoner R., Fowler W., Hoyle F. 1967 ApJ 148, 3

Wagoner R. 1969 ApJ supp. 18, 247

Wagoner R. 1973 ApJ 179, 343

Weymann R. 1965 Phys. Fluids 8, 2112

Weymann R. 1966 ApJ 145, 560

White M. et al. 2000 astro-ph/0008167 
Yang J. et al. 1979 ApJ 227, 697

Yang J. et al. 1984 ApJ 281, 493

Yoneyama T. 1972 Pub. Astr. Soc. Japan 24, 87

Zel'dovich Y., Kurt V., Sunyaev R. 1968, Zh. Eksp. Teor. Fiz. 55, 287

Zwicky F. 1959 PASP 71, 468

Zygelman B., Stancil P., Dalgarno A. 1998 ApJ 508, 151 